\title{
Management of a Spontaneous Thyroid Nodule Hemorrhage Causing Acute Airway Obstruction
}

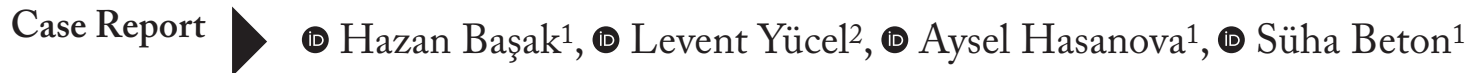 \\ ${ }^{1}$ Department of Otorhinolaryngology, Head and Neck Surgery, Ankara University School of Medicine, Ankara, Turkey \\ ${ }^{2}$ Department of Otorhinolaryngology, Head and Neck Surgery, University of Health Sciences Turkey, \\ Gülhane Research and Training Hospital, Ankara, Turkey
}

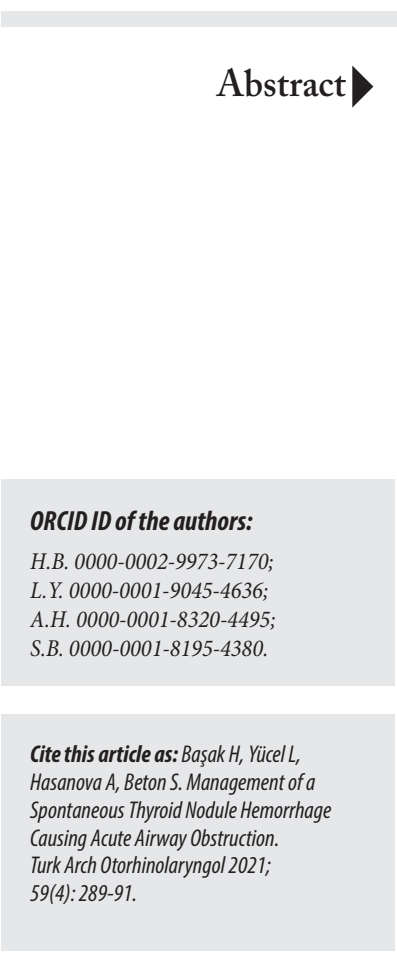

Corresponding Author:

Hazan Basak; hbasak@ankara.edu.tr

Received Date: 2021-05-31 Accepted Date: 2021-07-15

Content of this journal is licensed under a Creative Commons Attribution 4.0 International License. Available online at www.turkarchotolaryngol.net

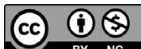

DOI: $10.4274 / \operatorname{ta0} .2021 .2021-5-23$

Spontaneous intrathyroidal hemorrhage (ITH) causing airway obstruction is relatively rare. We report a case with no known history of a thyroid disease that presented with an ITH causing severe airway obstruction as a life-threatening situation. A 57 -year-old female patient presented to the emergency department with sudden onset of swelling of the anterior neck, severe dyspnea, and unconsciousness. Computerized tomography scan after intubation revealed a $6.2 \times 5.3 \mathrm{~cm}$ mass originating from the right thyroid lobe and compressing the airway. Drainage of hematoma with right lobectomy was done. When the patient was extubated one day after the surgery the vocal cords were found to be mobile. She was discharged after two days and there was no need for further intervention during the one-month follow-up. Spontaneous life-threatening ITHs are rare entities. Immediate assessment of airway obstruction and achieving a secure airway are crucial. Besides drainage of hematoma, thyroidectomy may also be necessary.

Keywords: Airway obstruction, thyroidectomy, goiter, hematoma, spontaneous hemorrhage, emergency, case report

\section{Introduction}

Thyroid gland hemorrhage is a lifethreatening situation if not managed promptly and correctly. Spontaneous intrathyroidal hemorrhage (ITH) causing airway obstruction is relatively rare and different causes are reported in the literature (1-4). Spontaneous hemorrhage of the thyroid gland usually occurs in cases with thyroid nodules and cysts; however, the reason for rupture is still not clear (2, 4-6). We report a case with no known history of a thyroid disease that presented with an ITH causing severe airway obstruction as a life-threatening situation.

\section{Case Presentation}

A 57-year-old female patient presented to the emergency department with sudden onset of swelling of the anterior neck, severe dyspnea, and unconsciousness. The patient was intubated to secure the airway and referred to our department. The larynx had shifted to the left however; the vocal cords were visible. She had no history of any disease. According to her relatives' statement, dizziness and nausea had started 12 hours ago and swelling began to appear on the right side of her neck in the meantime. She had forced herself to vomit to feel better and then slept. After 
few hours, her relatives were unable to wake her up fully. The size of the swelling was rapidly increasing, and dysphagia and severe dyspnea ensued the enlargement of the swelling. They brought her to the emergency service.

Blood gas analysis and computerized tomography (CT) of the neck were done in emergency setting. Blood gas analysis revealed severe acidosis with a $\mathrm{pH}$ of 6.3. Right after securing the airway, the patient was transported to the intensive care unit and treatment of acidosis was administered. CT scan revealed a $6.2 \times 5.3 \mathrm{~cm}$ mass originating from the right thyroid lobe and compressing the trachea; and rupture of a cystic thyroid nodule was suspected (Figure 1).

An urgent surgery for drainage of the hematoma was decided because of tracheal compression, active bleeding, and rapid enlargement of the size of hematoma. Intraoperatively, a huge mass was seen to compress the trachea and shift it to the left side. Careful sharp dissection with coagulation was performed and blood clots were removed from inside the cyst. However, there was still bleeding in the surgical area and despite massive cauterization, there was a risk of a recurrent hematoma. Thus, the removal of the right lobe of the thyroid was deemed necessary. After identifying and preserving the recurrent laryngeal nerve (RLN) and parathyroid glands, lobectomy was performed (Figure 2). The patient was not extubated immediately after the operation, but on the next day; and the vocal cord movements and thyroid function tests were normal. After two days, she was discharged with oral antibiotics. During the one-month follow-up there was no need for further intervention. Histopathologic report revealed a multinodular goiter with a hemorrhage of cystic nodule. The patient was referred to the endocrinology department for further follow-up.

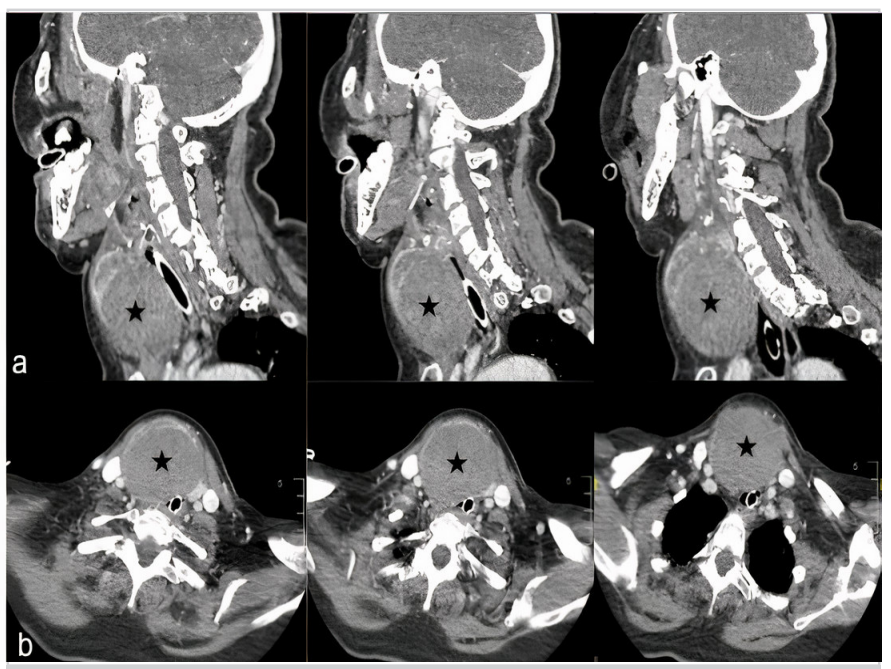

Figure 1. Computerized tomography scan showing a. Sagittal sections: a huge hematoma compressing the airway b. Axial sections: hematoma arising from the right thyroid lobe compressing the trachea and causing a shift to the left side. $(\star)$ indicates right thyroid lobe

\section{Discussion}

ITH is relatively rare and the mechanism causing the hemorrhage is unknown. Some of the factors reported in the literature may be grouped as traumatic and nontraumatic causes $(1,2,4,5)$. Blunt trauma to the anterior neck region or fine needle aspiration biopsy are responsible for most of the cases. Use of anticoagulants, bleeding diathesis are other reasons for ITH. Even though increased intravascular pressure such as Valsalva maneuver, choking, constipation could be accepted as the etiological factors of spontaneous ITH, there is no consensus as to why hemorrhage occurs (3). Increased vascularization of the gland and more specifically arteriovenous shunting inside the nodule are thought to be the causal factors $(2,4,6,7)$. In the presented case, increased intravascular pressure caused by vomiting could be the reason for ITH. Yang et al. (2) found that abundant blood supply and spongiform internal content of the nodule revealed by ultrasound were associated with ITH of their patients with partial cystic thyroid nodule.

The symptomatology of ITH ranged from asymptomatic to life-threatening airway compromise. Dysphagia, swelling of the anterior neck, dyspnea are the main symptoms of ITH depending on the size of the hematoma (2). The overall condition and vital parameters of the patient must be assessed before any radiological imaging. Flexible nasolaryngoscopy of those with symptoms is the fastest way to evaluate the

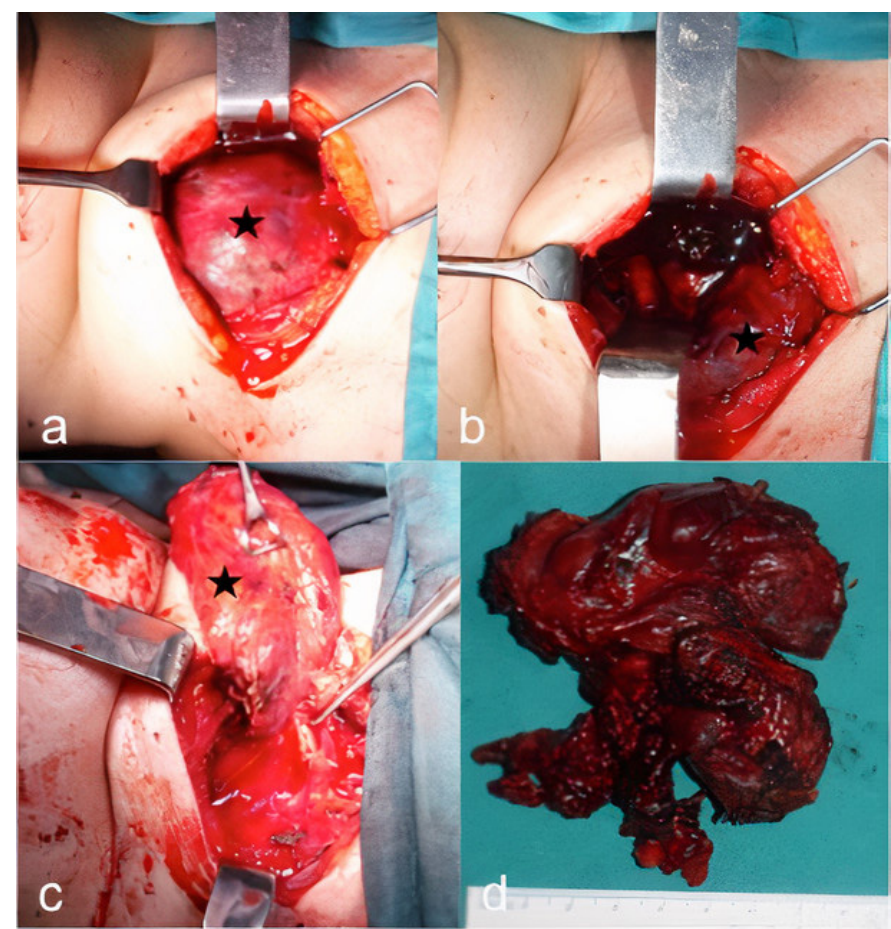

Figure 2. a. Right thyroid lobe b. Hematoma arising from right thyroid lobe c. Identifying the recurrent laryngeal nerve and view of the right thyroid lobe intranodular vessels d. Specimen. ( $\star$ indicates right thyroid lobe) 
airway. An urgent neck CT with contrast or ultrasound can show the size of hematoma and enable to assess airway obstruction (2, 4, 5, 8-10). However, as with our case, it may not be possible to wait for a CT scan and immediate intubation becomes of vital importance.

The most critical stage in the management of ITH is ensuring a secure airway. If intubation is not performed in a prompt and timely manner, airway impairment may occur rapidly, severe morbidities may develop, and emergency intervention may be needed. Venous congestion and edema may cause dyspnea in case of active bleeding even when the airway is not compressed.

In most cases, patients with ITH do not need an urgent thyroidectomy. After urgent drainage of hematoma, elective thyroidectomy with neuromonitoring guidance will be the best option $(2,6,7,9,10)$. In some cases, however, it can be challenging to stop the bleeding and an urgent subtotal thyroidectomy can become necessary. In our case, right after surgical drainage, it was not possible to stop bleeding from the thyroid tissue and we decided to perform a lobectomy in the same session. Although we prefer to perform all kinds of thyroid surgeries under neuromonitoring guidance, this method was not possible in this case given the high risk of reintubation failure with a neuromonitoring endotracheal tube. Subtotal thyroidectomy may be an option to reduce the risk of re-bleeding; however, it should be kept in mind that benign nodules can also cause ITH as well as malignant nodules $(6,7)$. We preferred lobectomy for this case because we did not have a clear sign that the patient had a kind of a malignant thyroid disease.

ITH with mild symptoms can be followed with serial ultrasound examinations, administering steroid and antibiotic therapy to prevent edema and abscess formation (5). Cases with ITH due to anticoagulant therapy are usually treated with conservative methods. It is associated with nodular thyroid disease and generally suspension of anticoagulant therapy and changing treatment to low molecular weight heparin helps to avoid worsening of symptoms $(2,4)$.

\section{Conclusion}

Spontaneous life-threatening ITH is a rare entity. Immediate assessment of airway obstruction and achieving a secure airway are crucial. If bleeding cannot be stopped after drainage or surgical exploration, thyroidectomy can become imperative in the same session.

Informed Consent: This is a retrospective case study.

Peer-review: Externally peer-reviewed.

\section{Author contributions}

Conception: H.B., L.Y., Design: H.B., A.H., Supervision: S.B., Data Collection and/or Processing: H.B., L.Y., A.H., Analysis and/or Interpretation: H.B., L.Y., Literature
Review: H.B., L.Y., A.H., S.B., Writing: H.B., Critical Review: H.B., L.Y., S.B.

Conflict of Interest: No conflict of interest was declared by the authors.

Financial Disclosure: The authors declared that this study received no financial support

\section{Main Points}

- Thyroid gland hemorrhage may be a life-threatening situation if not managed promptly and correctly.

- Increased vascularization of the gland and more specifically arteriovenous shunting inside the nodule and abundant blood supply are the factors thought to be the underlying reasons.

- The most critical stage in the management of ITH is ensuring a secure airway.

- In most cases, urgent thyroidectomy is not needed.

- In cases when bleeding cannot be controlled with drainage and surgical exploration, thyroidectomy may become imperative.

\section{References}

1. Petersen NK, Udholm S, Londero SC. Acute airway obstruction due to spontaneous intrathyroidal haemorrhage. BMJ Case Rep 2021; 14: e238935. [Crossref]

2. Yang H, Zhao S, Zhang Z, Chen Y, Wang K, Shang M, et al. The associated factors for spontaneous intranodular hemorrhage of partially cystic thyroid nodules: a retrospective study of 101 thyroid nodules. Medicine 2020; 99: e23846. [Crossref]

3. Covino M, Princi P, De Luca G, Del Ciello A, Simeoni B, Bianchi A, et al. Spontaneous thyroid nodule hemorrhage in the emergency department. Endocr Pract 2020; 26: 192-6. [Crossref]

4. Gunasekaran K, Rudd KM, Murthi S, Kaatz S, Lone N. Spontaneous thyroid hemorrhage on chronic anticoagulation therapy. Clin Pract 2017; 7: 36-8. [Crossref]

5. Zhu T, Yang Y, Ju H, Huang Y. Acute thyroid swelling after fine needle aspiration - a case report of a rare complication and a systematic review. BMC Surg 2021; 21: 175. [Crossref]

6. Liang TJ, Liu SI. Spontaneous bleeding from a thyroid adenoma. Ear Nose Throat J 2016; 95: 268-73. [Crossref]

7. Vijendren A, Ladha N, Hilgar A. Airway emergency from spontaneous haemorrhagic thyroid cancer. Med J Malaysia 2014; 69: 187-8. [Crossref]

8. Giotakis EI, Hildenbrand T, Dodenhöft J. Sudden massive neck swelling due to hemorrhage of a thyroid adenoma: a case report. J Med Case Rep 2011; 5: 391. [Crossref]

9. Pompeo A, Staniscia G, De Carlo F. [An acute superior mediastinal syndrome with critical tracheal stenosis due to benign multinodular goiter complicated by intracystic hemorrhage.] Ann Ital Chir 1999; 70: 589-92. [Crossref]

10. Wong M, Kanapaty Y, Liew YT, Bakri A. Spontaneous thyroid haemorrhage: a cause of upper airway obstruction. Acute Med 2019; 18: 248-50. [Crossref] 